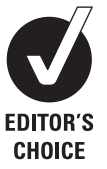

Department of Children and Adolescents, Oulu University Hospital, Oulu, Finland ${ }^{2}$ Oulu City Health Care Centre, Oulu, Finland

${ }^{3}$ Hospital for Children and Adolescents, University of Helsinki, Helsinki, Finland ${ }^{4}$ Department of Paediatrics, Tampere University Hospital, Tampere, Finland

${ }^{5}$ Department of Paediatrics, Kuopio University Hospital,

Kuopio, Finland

${ }^{6}$ Department of Paediatrics, Helsinki University Central Hospital, Espoo, Finland

${ }^{7}$ Department of Paediatrics, Hyvinkää Hospital, Hyvinkää, Finland

\section{Corresponding to}

Matti Nuutinen, Department of Children and Adolescents, Oulu University Hospital, P.O Box 23, OYS (Oulu) 90029, Finland; matti.nuutinen@ ppshp.fi

Accepted 22 December 2009

\title{
Clinical course of extrarenal symptoms in Henoch-Schönlein purpura: a 6-month prospective study
}

\author{
Outi Jauhola, ${ }^{1}$ Jaana Ronkainen, ${ }^{2}$ Olli Koskimies, ${ }^{3}$ Marja Ala-Houhala, ${ }_{1}^{4}$ Pekka Arikoski, ${ }^{5}$ \\ Tuula Hölttä, ${ }^{3}$ Timo Jahnukainen, ${ }^{3}$ Jukka Rajantie, ${ }^{6}$ Timo Örmälä, Matti Nuutinen ${ }^{7}$
}

\begin{abstract}
Objective To describe the extrarenal symptoms and clinical course of Henoch-Schönlein purpura (HSP).

Design A prospective national multicentre trial with 6-month follow-up.
\end{abstract}

Patients A total of 223 newly diagnosed paediatric HSP patients.

Results Purpura was the initial symptom in $73 \%$ of the patients and was preceded by joint or gastrointestinal manifestations in the rest by a mean of 4 days. Joint symptoms, abdominal pain, melena, nephritis and recurrences occurred in $90 \%, 57 \%, 8 \%, 46 \%$ and $25 \%$ of the patients, respectively. Orchitis affected 17/122 (14\%) of the boys. Seven patients developed protein-losing enteropathy characterised by abdominal pain, oedema and serum albumin under $30 \mathrm{~g} / \mathrm{l}$, and an additional 49 patients had subnormal albumin levels without any proteinuria. Positive fecal occult blood (26/117, 22\%) and $\alpha 1$-antitrypsin $(7 / 77,9 \%)$ suggested mucosal injury even in the patients without gastrointestinal symptoms. HSP was often preceded by various bacterial, especially streptococcal (36\%) and viral infections. Previous streptococcal infection did not induce changes in the level of complement component C3. Recurrences were more frequent in patients $>8$ years of age $(\mathrm{OR} 3.7, \mathrm{Cl}$ 2.0 to $7.0, p<0.001)$ and in patients with nephritis (OR 4.6, Cl 2.3 to 8.9, $p<0.001)$. Patients with severe HSP nephritis had more extrarenal symptoms up to 6 months. There was no difference in the clinical course between the prednisone-treated and non-treated patients during the 6-month follow-up.

Conclusions Serum albumin is often low in HSP patients without proteinuria, due to protein loss via the intestine. Although corticosteroids alleviate the symptoms, they seem not to alter the clinical course of HSP during 6 months of follow-up.

\section{INTRODUCTION}

Henoch-Schönlein purpura (HSP) is the most common form of small vessel vasculitis in children. A population-based study has shown the annual incidence to be 20.4 per 100000 in children $<17$ years. ${ }^{1}$ The dominant clinical features are petechiae, arthritis, abdominal pain and nephritis, but other organs such as the brain or lungs and the scrotum may occasionally be involved. Most paediatric patients have a self-limited disease, but severe abdominal pain, intestinal bleeding or intussusception may cause acute complications. ${ }^{2-4}$ The renal symptoms of HSP vary from intermittent haematuria and proteinuria to severe nephrotic-nephritic syndrome.

\section{What is already known on this topic}

- Corticosteroids alleviate the extrarenal symptoms of Henoch-Schönlein purpura (HSP) effectively, but do not prevent the development of nephritis.

- About $60 \%$ of the HSP patients have gastrointestinal symptoms typically manifested as colicky abdominal pain and sometimes melena.

\section{What this study adds}

- Corticosteroids did not alter the clinical course of extrarenal symptoms during the 6 -month follow-up and did not increase the rate of recurrences.

- Gastrointestinal involvement, measured by fecal occult blood and $\alpha 1$-antitrypsin, is frequent also in patients without any gastrointestinal symptoms and may cause intestinal protein loss and hypoalbuminaemia.

The prognosis for HSP is considered excellent in patients without renal disease. ${ }^{56}$ The pathogenesis is not completely understood, but exposures to various antigens such as infective agents, vaccinations and drugs are considered possible as immunological triggers for the vasculitis. ${ }^{4}$ Although HSP was first described over 200 years ago, there is a scarcity of data on its clinical outcome from large-scale prospective studies. Our data describe the clinical course of the extrarenal symptoms based on a 6-month follow-up.

\section{PATIENTS AND METHODS}

The criteria for inclusion were age $\leq 16$ years and a clinical diagnosis of HSP including typically distributed palpable purpura or petechiae and at least one of the following findings: arthralgia or arthritis, abdominal pain or nephritis. Exclusion criteria were thrombocytopenia and systemic vasculitis at onset. The diagnosis of HSP fulfils the 1990 criteria of the American College of Rheumatology. ${ }^{8}$

A total of 223 children, 122 boys and 101 girls, with newly diagnosed HSP were enrolled 
between October 1999 and February 2006. Most of the patients were primarily recruited into a trial on early corticosteroid treatment for the prevention of nephritis ${ }^{9}$ or one comparing cyclosporine A (CyA) with methylprednisolone (MP) pulse for treating severe HSP nephritis. ${ }^{10}$ In the first case patients were randomised to receive either a placebo or prednisone $1 \mathrm{mg} / \mathrm{kg}$ for 2 weeks followed by a weaning dose for another 2 weeks, and in the latter case they were given either CyA $5 \mathrm{mg} / \mathrm{kg}$ for 12 months or MP pulses 30 $\mathrm{mg} / \mathrm{kg}$ intravenously three times followed by prednisone $30 \mathrm{mg} / \mathrm{m}^{2}$ for 4 months. Severe nephritis was defined as the existence of nephrotic-range proteinuria or ISKDC grade III to VI in a kidney biopsy. In addition, we analysed the onset data of patients who had been diagnosed as having HSP but did not attend the randomised trials. The specific details of the patients and their treatments are set out in figure 1 . The

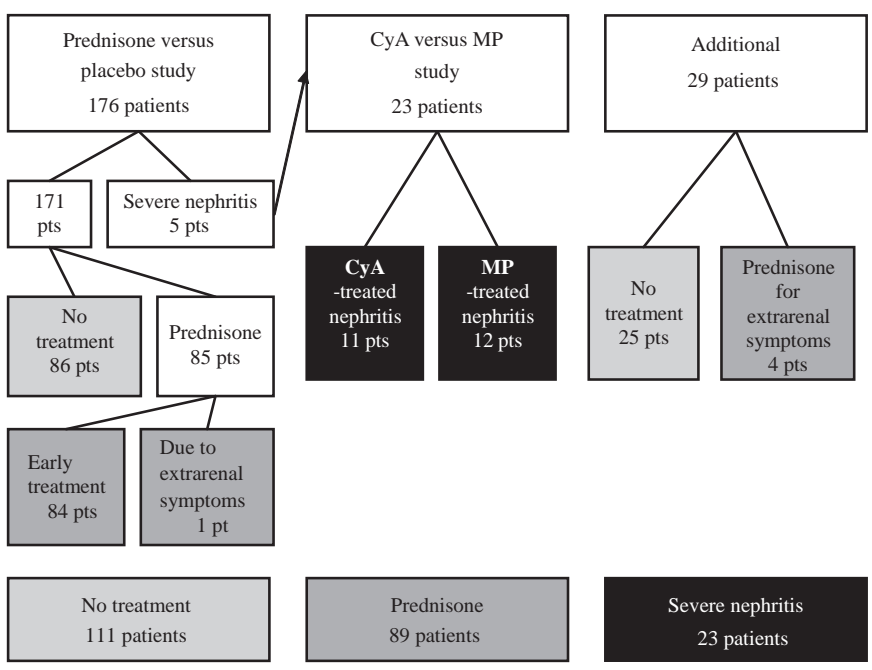

Figure 1 Numbers of patients taking part in the randomised prospective studies and the treatment they received for Henoch-Schönlein purpura.

trials had been approved by the local ethics committee in each centre, and written informed consent was obtained from all the parents.

The mean age of the patients at HSP onset was 7.1 years (SD 3.5, range 1.6-16.7 years), and 52\% were aged over 6 years. Males were affected slightly more frequently than females $(\mathrm{M}: \mathrm{F}$ ratio=1.2:1). There were no major differences between the boys and girls in the distribution of cases by age.

The patients were asked about the initial HSP symptoms and their duration upon inclusion, and attended five control visits during the 6-month follow-up (at inclusion and at 1-2 weeks, 1, 3 and 6 months after diagnosis). These visits included laboratory tests and a clinical examination by a paediatrician. The patients were also advised to contact their centre after the follow-up time for confirmation of any recurrence of HSP by a paediatric nephrologist. A recurrence was defined as an instance of a patient who had been asymptomatic for 1 month presenting with a new flare-up of skin lesions or other symptoms related to HSP.

The aetiology of HSP disease was explored in detail by eliciting an extensive medical history and performing specific laboratory tests. Infections preceding HSP onset by 6 months were recorded, and a full blood cell count, erythrocyte sedimentation rate (ESR), $\mathrm{C}$ reactive protein (CRP), serum albumin, complement component C3, fecal occult blood, $\alpha 1$-antitrypsin and urine analyses were performed at the time of diagnosis. To assess possible infectious triggers, we analysed a throat swab culture, antistreptolysin $\bigcirc$ titre, and streptodornase, teichoic acid and viral antibodies. National normal ranges for the patient's age and sex were used in the interpretation of the test results.

\section{Statistical analysis}

Continuous data were assessed in terms of means and standard deviations and categorical variables as percentages. Statistical analyses were carried out using the $\chi^{2}$ test for categorical variables and Student's two-tailed t test for continuous variables. Statistical significance was defined as $p<0.05$. All the statistical analyses were performed using the Statistical Package for Social Sciences (SPSS/Windows V.16.0, SPSS Inc., Chicago, Illinois, USA).

\section{RESULTS}

\section{Symptoms at presentation}

Petechiae were the first sign at presentation in 73\% (157/216) of the patients, while the skin lesions were preceded by joint symptoms $(33 / 216,15 \%)$, gastrointestinal symptoms $(23 / 216$, $11 \%)$ or both $(3 / 216,1 \%)$ by a mean of 4 days (SD 3.7, range 1-19 days) in the rest of the cases. The mean delay from the onset of the first symptoms to diagnosis at the hospital's paediatric outpatient clinic and inclusion in the study was 7 days (SD 10.5, range 0-65 days). Over $70 \%$ of the patients were diagnosed within 1 week of the occurrence of the first symptoms, while in eight cases the diagnosis was delayed for over 1 month.

\section{Joint and gastrointestinal symptoms of HSP}

All the HSP extrarenal symptoms were typically present at an early phase, although joint and abdominal symptoms could appear for the first time up to 1 month after the diagnosis (figure 2). Pain, swelling and functional limitation of the joint indicated joint involvement in 200/221 (90\%) of the patients during the follow-up, most frequently affecting the feet/ ankles (85\%) and knees (38\%). Symptoms in the upper limbs were less common, $29 \%$ having manifestations in the wrists and $14 \%$ in the elbows. A further six patients had articular impairment in the hips. The scrotum was affected by oedema in $17 / 122(14 \%)$ of the boys, all aged $2-9$ years.

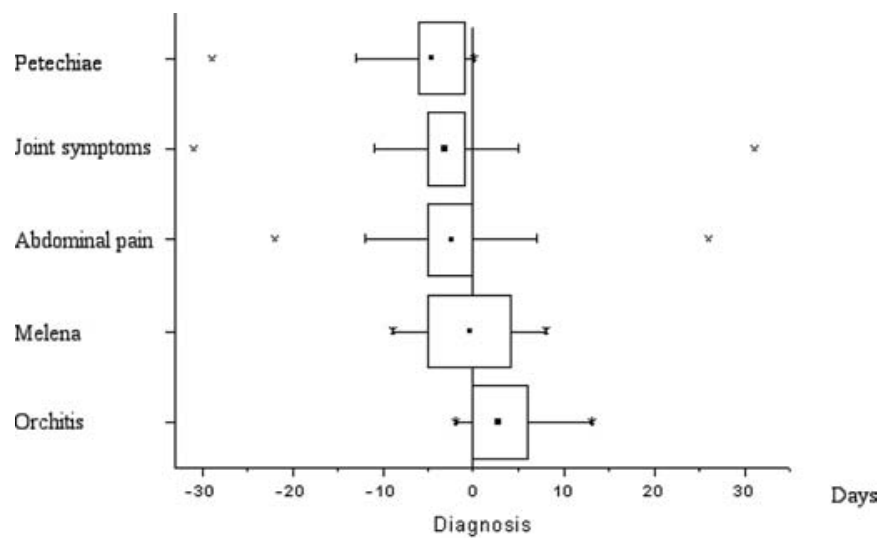

Figure 2 Timing of the appearance of extrarenal symptoms, in days, in 205 patients diagnosed with Henoch-Schönlein purpura within 1 month of the onset of the initial symptoms. Minimum and maximum values (X), mean values (closed square) and 5th, 25th, 75th and 95th percentiles are given. 
Gastrointestinal symptoms occurred in the form of abdominal pain in 126/221 cases (57\%). Of these 18 had gastrointestinal bleeding in the form of melena and two also in the form of haematemesis. These symptoms were short in duration and did not require any special treatment. The abdominal pain was considered severe in 17/126 cases (13\%), requiring admission to hospital for observation. The fecal occult blood and $\alpha 1$-antitrypsin tests performed at inclusion were positive in $22 \%(26 / 117)$ and $9 \%(7 / 77)$ of cases, respectively, suggesting mucosal injury. Of these patients only 10/26 (38\%) and 1/7 (14\%) had abdominal pain at the time of the test. A low serum albumin level (range 25-36.9 g/l) without any proteinuria was observed at inclusion in $44 / 179$ (25\%) of the patients, indicating protein leakage into the bowel, and an additional 12 patients developed hypoalbuminaemia without proteinuria later during the follow-up. Severe protein-losing enteropathy was observed in seven patients, being manifested in the form of abdominal pain, oedema and low serum albumin, ranging from 25 to $30 \mathrm{~g} / 1$.

\section{Clinical features during the 6-month follow-up}

The 111 patients who received no treatment describe the natural course of HSP as compared with the 89 patients treated with prednisone and the 23 with severe HSP glomerulonephritis. Altogether 202/223 (91\%) of the patients completed the 6 -month follow-up. The patients treated with prednisone had HSP symptoms less frequently during the first month after diagnosis, that is, at the time of medication, than did those who received no treatment (figure 3). The duration of abdominal and joint pain and the respective pain scores were lower in the prednisone-treated patients, as has been reported earlier. ${ }^{9}$ The difference was statistically significant in the prevalence of petechiae $(p=0.044)$ and any symptoms $(p=0.005)$ at the first control visit, but no difference was seen later during the follow-up. The patients with severe HSP nephritis also had extrarenal symptoms more frequently than did the patients with milder HSP disease for the whole 6-month follow-up time. The prevalence of petechiae, nephritis and any symptoms was statistically significantly higher at every control visit, with $p$ values ranging from $<0.001$ to 0.008 , and the prevalence of joint symptoms was higher at 1 month $(p=0.031)$.

Recurrences occurred in 55/221 cases (25\%), and 16 of these patients had several HSP episodes. Three patients had late recurrences 14, 18 and 26 months after the initial onset of the
Prevalence of petechiae

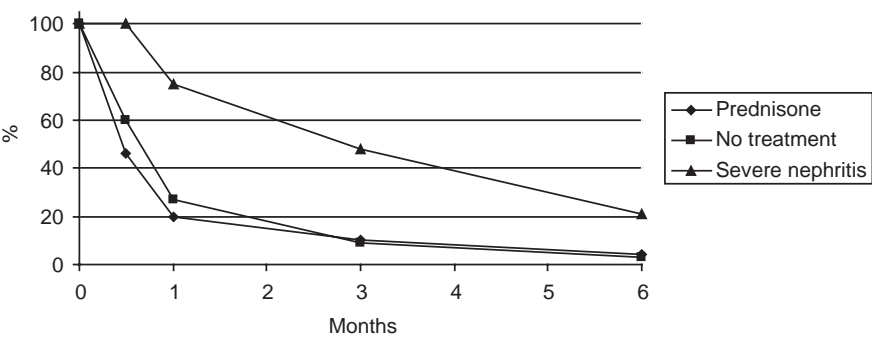

Prevalence of abdominal pain

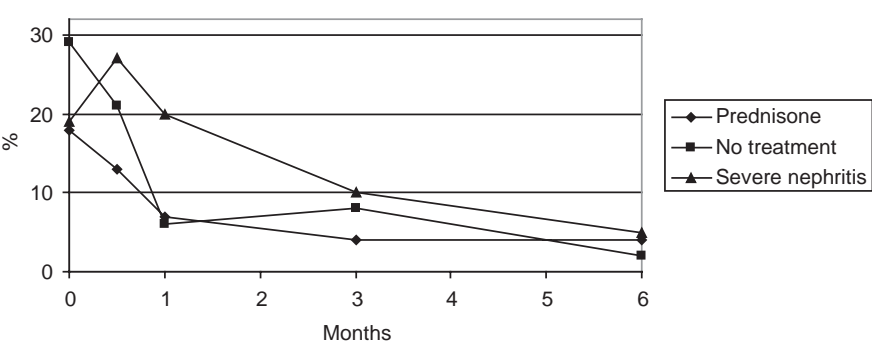

Prevalence of any symptoms

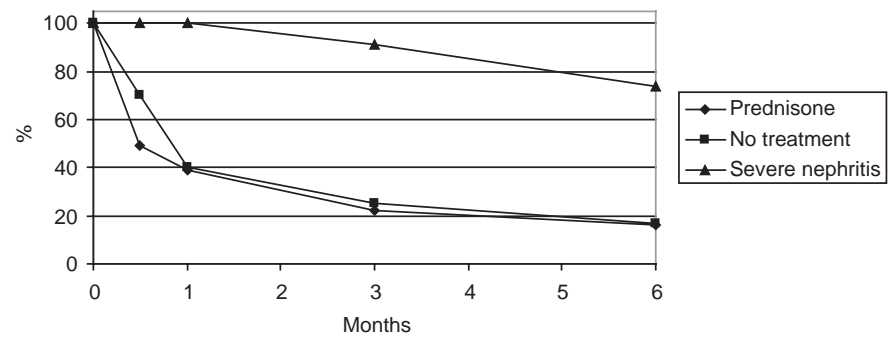

Prevalence of joint symptoms

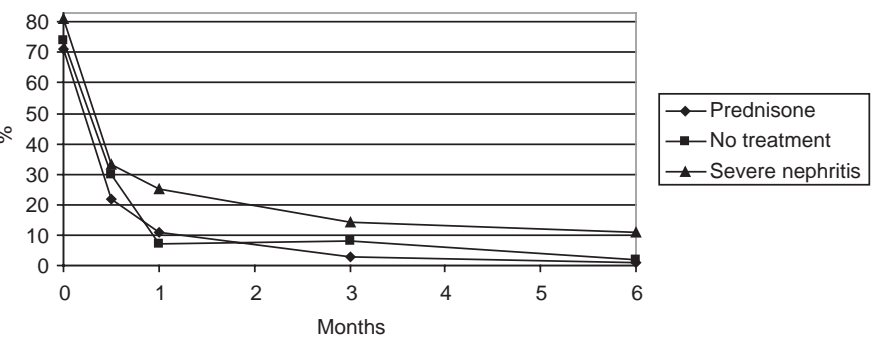

Prevalence of nephritis

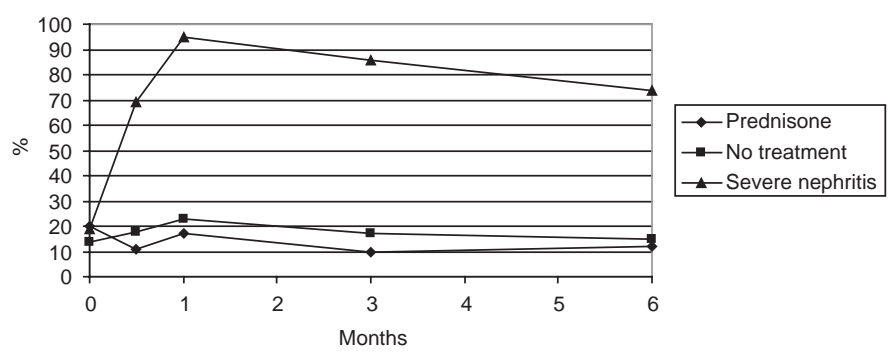

Figure 3 Clinical course of Henoch-Schönlein purpura in non-treated patients $(n=111)$, prednisone-treated patients $(n=89)$ and patients with severe glomerulonephritis $(n=23)$ during the 6-month follow-up. 
disease, as confirmed by a paediatric nephrologist at a control visit to the same centre. Recurrences occurred more frequently in patients over 8 years of age (OR 3.7, CI 2.0 to $7.0, \mathrm{p}<0.001)$ and in patients with nephritis (OR 4.6, CI 2.3 to 8.9, p<0.001). There was no difference in the recurrence rate within 6 months between the prednisone-treated $(22 / 89,25 \%)$ and non-treated groups $(19 / 111,17 \%, p=0.22)$. No intestinal intussusceptions occurred.

\section{Aetiology}

Signs of streptococcal infection were found in $71 / 199$ (36\%) of the patients, the throat swab culture being positive for group A $\beta$-haemolytic streptococcus in 50/165 (30\%) cases, and the antistreptolysin $\bigcirc$ titre or streptodornase antibodies positive in 19/159 (12\%) and 41/140 (30\%), respectively. Teichoic acid antibodies, implying a previous staphylococcal infection, were elevated in only one patient. Antibodies to parainfluenzae, adenovirus, $C M V$, influenzae $A$, mycoplasma, enterovirus, $R S V$, coxsackie or influenzae $B$ viruses were found in $89 / 149$ (60\%) patients. No systematic second antibody titre was taken. HSP had been preceded by an upper respiratory tract infection (URTI) in $72 \%$ $(n=161)$ of patients, in $53 \%(n=86)$ of whom it had occurred within 2 weeks prior to HSP onset. Tonsillitis, gastroenteritis or other febrile infections prior to HSP onset were also observed. At the acute phase of HSP, 93/181 (51\%) had elevated ESR (mean 30, SD 17.7, range 16-103 mm/h), 77/206 (37\%) elevated CRP (mean 30, SD 25.3, range 11-158 mg/l) and 12/213 (6\%) leucocytosis (mean 17, SD 2.9, range 14-24 10e9/1), possibly indicating a previous infection. Elevated ESR was common in patients with protein leakage into the bowel, occurring in $27 / 38(71 \%)$ of these cases. HSP occurred more commonly at the coldest time of the year $(78 \%$ diagnosed between September and March). The level of complement component C3 was increased in 10/175 (6\%), showing no association with previous streptococcal infection.

\section{DISCUSSION}

To our knowledge this is the largest prospective study of childhood HSP describing the clinical course of the disease based on a 6-month follow-up of 223 unselected patients. The mean age of 7.1 years, and the slight predominance of boys, $55 \%$, are consistent with previously published results. Our comparative analysis of the HSP clinical features (table 1) shows that the ranges of variation between individual studies are quite large. In some cases the patient series may be selected, or even misdiagnosed, as not all the HSP patients are reported to have had petechiae, or else no other symptoms were observed at presentation other than petechiae. The mean prevalence of joint symptoms ( $91 \%$ vs $68 \%$ ) and recurrences ( $36 \%$ vs $17 \%$ ) were higher in the prospective studies possibly on account of recall bias in the retrospective studies.

The proportion of our patients admitted to hospital for observation because of severe abdominal pain was $13 \%$, while $8 \%$ had gastrointestinal bleeding, but no severe gastrointestinal complications occurred. It has been suggested that steroid therapy may reduce the risk of intussusception, ${ }^{11}$ but this complication is in any case rare in HSP. ${ }^{4} 12$ Melena is thought to occur only in HSP patients with severe abdominal pain, ${ }^{34} 13$ as also noted here. Our results of positive fecal occult blood and $\alpha 1$-antitrypsin in patients without abdominal pain suggest mucosal injury even in patients without gastrointestinal symptoms. Protein-losing enteropathy, a rather rare feature of HSP, occurred in seven of our patients. In addition, 49 patients had a subnormal serum albumin level without renal loss, this occurring most frequently at the onset of the disease. Such a finding was frequently associated with an elevated ESR value. Hypoalbuminaemia in HSP patients is generally caused by HSP nephritis-related proteinuria. The absence of significant proteinuria in a patient with hypoalbuminaemia should arouse a suspicion of intestinal protein loss. It is therefore important to assay serum albumin in patients without any proteinuria, and even without gastrointestinal symptoms. Our results underline the need for proper, regular documentation of proteinuria and serum albumin in HSP patients.

The extrarenal symptoms of HSP are reported to be self-limiting within 3-8 weeks, while recurrences beyond 4 months are rare. ${ }^{2-4}$ The frequency of recurrences in our study was similar to the figures reported in the literature (table 1), while three patients had late recurrences up to 2 years after the initial HSP episode. The highest incidence of recurrences, $66 \%$, reported by Fretzayas et al in a prospective study of 74 patients, is possibly explained by their definition of recurrence with a larger scale of signs and symptoms and shorter asymptomatic period of 2 weeks. ${ }^{13}$ The clinical manifestations in each treatment group during the follow-up are described in figure 3 . It is interesting that $17 \%$ of the non-treated patients, $16 \%$ of the prednisone-treated patients and $74 \%$ of the patients with severe HSP nephritis still had symptoms related to HSP at the 6-month control visit.

Recurrences occurred more frequently in the patients with nephritis (OR 3.7) and those over 8 years of age (OR 4.6). Trapani et al reported in their retrospective study that recurrences correlated with high ESR values and corticosteroid therapy, $13 \%$ of their patients having received corticosteroids due to severe abdominal pain or nephropathy. ${ }^{3}$ The association between corticosteroid therapy and HSP recurrences was not confirmed in our study with randomised early corticosteroid therapy. Nor did we find any association between elevated ESR and recurrences. We would prefer to think of a severe disease at onset as constituting a major risk factor for recurrences rather than the treatment as such.

The majority of our patients had potential trigger events before the onset of HSP, including previous infections. On the other hand, this may only depict a normal course of life. The incidence of URTI before HSP onset is $36-63 \%$ in the literature $^{613-16}$ and was even higher in our patients, $73 \%$. Nong et al reported patients without previous URTI as having a higher incidence of gastrointestinal problems, ${ }^{17}$ and GonzalezGay et al noted that patients with previous URTI had a higher frequency of nephritis. ${ }^{16}$ We found no association between a history of URTI and the clinical picture of HSP, however.

Our data support the notion of an infectious trigger in the pathogenesis of HSP, but as pointed out by Saulsbury, ${ }^{18}$ no single pathogen appears to be the dominant precipitating cause of HSP. Among our patients, 122/201 (61\%) had positive serological tests or microbiological cultures at the time of the diagnosis. The most common pathogen, streptococcus, was observed in $71 / 122(58 \%)$ of these. We observed elevation in various viral antibodies, but the lack of systematically taken control serum samples complicates the interpretation, since elevated levels may merely indicate old-standing immunity. The elevated ESR and CRP levels, however, may mean that a recent infection is implicated. The role of streptococcus in the pathogenesis of HSP is debatable, ${ }^{18}$ although this is supported by the results of Masuda et al who described the presence of group A streptococcal antigen in the glomeruli of children with HSP nephritis. ${ }^{19}$ Streptococcal infection did not induce changes in C3. 


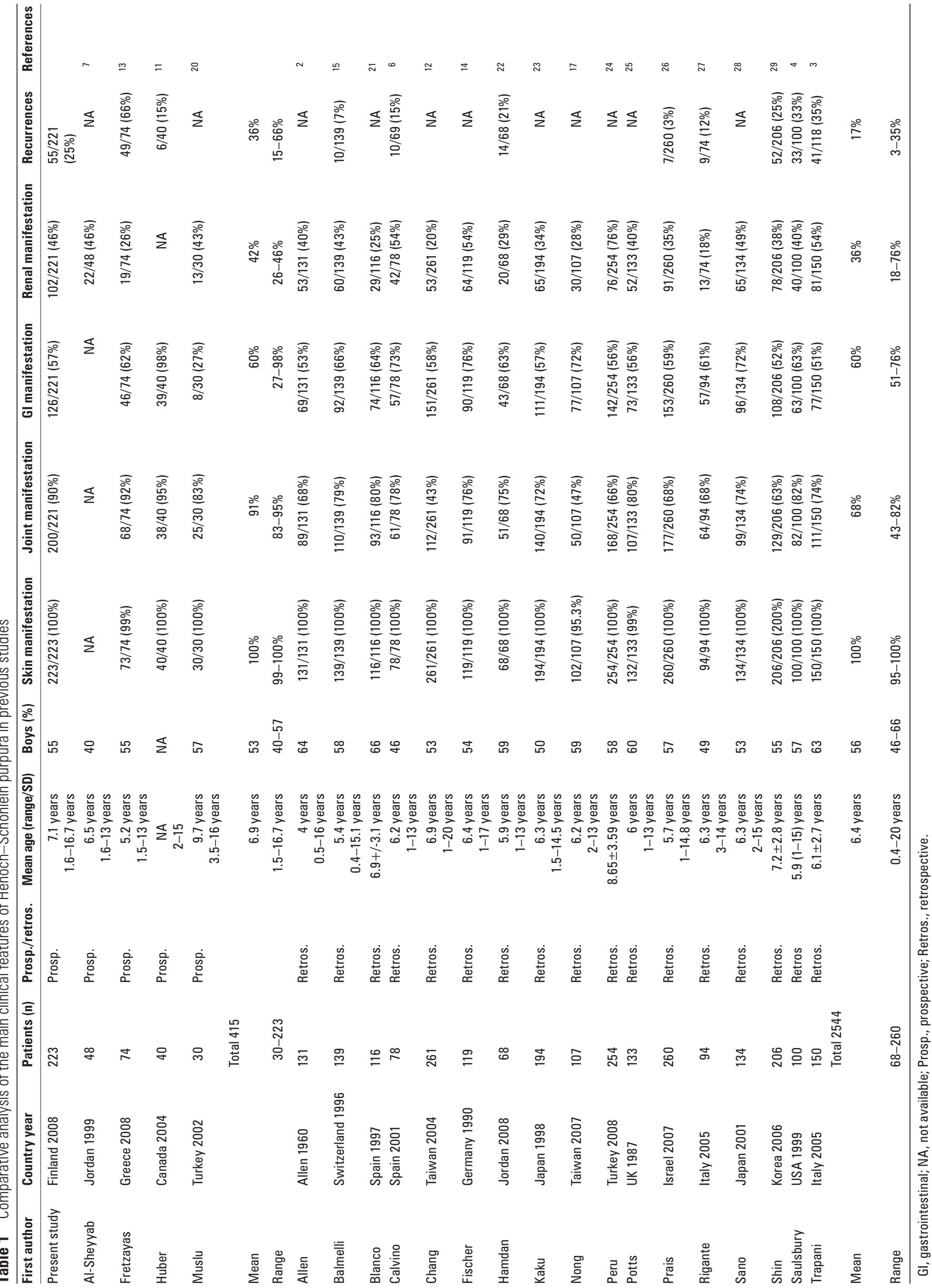


The role of corticosteroids in the treatment of HSP has been debated. ${ }^{911}$ HSP-related symptoms were slightly less frequent at the beginning in the prednisone-treated group than in the non-treated group (figure 3), but with a statistical difference only in the existence of petechiae at the 1-2-week control visit, and there were no differences between the two groups later in the follow-up. We have reported previously that early prednisone did not prevent the development of nephritis, although it did treat all the symptoms of HSP effectively. ${ }^{9}$ Our present results demonstrate that corticosteroid treatment did not alter the clinical course of HSP over 6 months. This finding is in accordance with our earlier conclusion that corticosteroids should not be used routinely but individually, in cases of severe HSP symptoms.

\section{CONCLUSIONS}

HSP is often preceded by various bacterial, especially streptococcal, and viral infections, although this may just reflect normal life. The serum albumin level should be monitored, because protein loss via the intestine is common in addition to proteinuria. The patients with severe HSP nephritis had extrarenal symptoms more frequently during the 6-month followup. There was no difference in the clinical picture after the acute phase between the prednisone-treated and non-treated patients, suggesting that corticosteroids do not alter the clinical course of HSP.

Funding $\mathrm{OJ}$ and JR received a grant from the Alma and K A Snellman Foundation, Oulu, Finland and from the Foundation for Paediatric Research for writing this report.

Competing interests None.

Provenance and peer review Not commissioned; externally peer reviewed.

Ethics approval This study was conducted with the approval of the ethics committees of each study centre (the university hospitals of Helsinki, Kuopio, Oulu, Tampere and Turku).

Patient consent Obtained.

\section{REFERENCES}

1. Gardner-Medwin JM, Dolezalova P, Cummins C, et al. Incidence of HenochSchönlein purpura, Kawasaki disease, and rare vasculitides in children of different ethnic origins. Lancet 2002;360:1197-202.

2. Allen DM, Diamond LK, Howell DA. Anaphylactoid purpura in children (Schonlein-Henoch syndrome). Am J Dis Child 1960;99:833-54.

3. Trapani S, Micheli A, Grisolia F, et al. Henoch Schonlein purpura in childhood: epidemiological and clinical analysis of 150 cases over a 5-year period and review of literature. Semin Arthritis Rheum 2005;35:143-53.

4. Saulsbury FT. Henoch-Schönlein purpura in children. Report of 100 patients and review of the literature. Medicine (Baltimore) 1999;78:395-409.

5. Ronkainen J, Nuutinen M, Koskimies 0 . The adult kidney 24 years after childhood Henoch-Schönlein purpura: a retrospective cohort study. Lancet 2002;360:666-70.

6. Calviño MC, Llorca J, García-Porrúa C, et al. Henoch-Schönlein purpura in children from northwestern Spain: a 20 -year epidemiologic and clinical study. Medicine (Baltimore) 2001;80:279-90.
7. al-Sheyyab M, Batieha A, el-Shanti $\mathrm{H}$, et al. Henoch-Schonlein purpura and streptococcal infection: a prospective case-control study. Ann Trop Paediatr 1999:19:253-5.

8. Mills JA, Michel BA, Bloch DA, et al. The American College of Rheumatology 1990 criteria for the classification of Henoch-Schönlein purpura. Arthritis Rheum 1990;33:1114-21.

9. Ronkainen J, Koskimies 0, Ala-Houhala M, et al. Early prednisone therapy in Henoch-Schönlein purpura: a randomized, double-blind, placebo-controlled trial. J Pediatr 2006;149:241-7.

10. Jauhola 0, Ronkainen J, Ala-Houhala M, et al. Cyclosporine A (CyA) versus MP pulses (MP) in severe Henoch-Schönlein nephritis (HSN): outcome after 2 year follow-up. Pediatr Nephrol 2008;23:1584

11. Huber AM, King J, McLaine P, et al. A randomized, placebo-controlled trial of prednisone in early Henoch Schönlein Purpura [ISRCTN85109383]. BMC Med 2004;2:7.

12. Chang WL, Yang YH, Lin YT, et al. Gastrointestinal manifestations in Henoch-Schönlein purpura: a review of 261 patients. Acta Paediatr 2004; 93:1427-31.

13. Fretzayas A, Sionti I, Moustaki M, et al. Henoch-Schönlein purpura: a long-term prospective study in Greek children. J Clin Rheumatol 2008;14:324-31.

14. Fischer PJ, Hagge W, Hecker W. [Schönlein-Henoch purpura. A clinical study of 119 patients with special reference to unusual complications]. Monatsschr Kinderheilkd 1990;138:128-34.

15. Balmelli C, Laux-End R, Di Rocco D, et al. [Schoenlein-Henoch purpura: course in 139 children]. Schweiz Med Wochenschr 1996;126:293-8.

16. Gonzalez-Gay MA, Calviño MC, Vazquez-Lopez ME, et al. Implications of upper respiratory tract infections and drugs in the clinical spectrum of HenochSchönlein purpura in children. Clin Exp Rheumato/ 2004;22:781-4.

17. Nong BR, Huang YF, Chuang CM, et al. Fifteen-year experience of children with Henoch-Schönlein purpura in southern Taiwan, 1991-2005. J Microbiol Immunol Infect 2007:40:371-6.

18. Saulsbury F. Epidemiology of Henoch-Schönlein purpura. Cleve Clin J Med 2002;11:87-9

19. Masuda M, Nakanishi K, Yoshizawa N, et al. Group A streptococcal antigen in the glomeruli of children with Henoch-Schönlein nephritis. Am J Kidney Dis 2003;41:366-70.

20. Muslu A, Islek I, Gok F, et al. Endothelin levels in Henoch-Schonlein purpura. Pediatr Nephrol 2002;17:920-5.

21. Blanco R, Martínez-Taboada VM, Rodríguez-Valverde V, et al. Henoch-Schönlein purpura in adulthood and childhood: two different expressions of the same syndrome. Arthritis Rheum 1997;40:859-64.

22. Hamdan JMM, Barqawi MA. Henoch-Schonlein purpura in children. Influence of age on the incidence of nephritis and arthritis. Saudi Med J 2008;29:549-52.

23. Kaku Y, Nohara K, Honda S. Renal involvement in Henoch-Schönlein purpura: a multivariate analysis of prognostic factors. Kidney Int 1998;53:1755-9.

24. Peru H, Soylemezoglu 0, Bakkaloglu SA, et al. Henoch Schonlein purpura in childhood: clinical analysis of 254 cases over a 3-year period. Clin Rheumatol 2008;27:1087-92.

25. Potts SR, Hamilton JR, Stewart M, et al. Henoch-Schönlein purpura: problems in surgical diagnosis and management. Ulster Med $\mathrm{J}$ 1987:56:100-3.

26. Prais D, Amir J, Nussinovitch M. Recurrent Henoch-Schönlein purpura in children. J Clin Rheumatol 2007;13:25-8.

27. Rigante D, Candelli M, Federico G, et al. Predictive factors of renal involvement or relapsing disease in children with Henoch-Schönlein purpura. Rheumatol Int 2005;25:45-8.

28. Sano H, Izumida M, Shimizu H, et al. Risk factors of renal involvement and significant proteinuria in Henoch-Schönlein purpura. Eur J Pediatr 2002;161:196-201.

29. Shin JI, Park JM, Shin YH, et al. Predictive factors for nephritis, relapse, and significant proteinuria in childhood Henoch-Schönlein purpura. Scand J Rheumatol 2006;35:56-60. 